\title{
A case of late onset steatocystoma multiplex
}

\author{
Meng Jiang, Mengli Zhang, Heng Gu, Xu Chen
}

Institute of Dermatology, Jiangsu Key Laboratory of Molecular Biology for Skin Diseases and STIs, Chinese Academy of Medical Science and Peking Union Medical College, Nanjing, China

A 74-year-old woman presented with multiple nodules on her scalp which were asymptomatic and discovered incidentally 10 years previously. The patient did not receive any treatment since there had been no subjective symptoms. Over time, however, the lesions increased gradually both in size and number. The patient was systemically healthy and there was no history of hypertension, diabetes, hyperlipidemia or similar lesions in her family. Dermatological examination showed that multiple white and yellow cystic nodules were discretely distributed over her scalp. These nodules were dome-shaped, smooth-surfaced, well defined and varied in size from 5 to $10 \mathrm{~mm}$ in diameter. The lesions were slightly hard and confined to the scalp, with no inflammation around the nodules. No other lesions could be seen around the body and no abnormality could be found in her nail, teeth or hair (Figure 1). Histopathological examination from one of the nodules demonstrated multiple epithelial cysts in the dermis. The folded cystic wall was lined with stratified squamous epithelium without a granular layer. Sebaceous glands were present in the cyst wall (Figure 2). Based on the clinical and histopathological features, a diagnosis of steatocystoma multiplex was made. No treatment was performed because of the benign process of this disease and asymptomatic character of the lesions. She was followed up for 1 year and showed no improvement in the involved lesions.

Steatocystoma multiplex is an autosomal dominant genodermatosis characterized by the presence of multiple sebum-containing dermal cysts. It is regarded as a nevoid or hamartomatous malformation of the pilosebaceous duct junction [1, 2]. Steatocystoma multiplex tends to occur in adolescents or early adulthood with no predilection for sex. Clinically, steatocystoma multiplex presents as multiple, smooth, dome-shaped, skin-colored or yellowish cystic nodules of various sizes. The nodules are firm and asymptomatic. The prominent histopathological features of steatocystoma multiplex are dermal cysts composed of thin walls of stratified squamous epithelium without a granular layer. The inner surface of the cyst wall shows a wavy appearance lined with homogenized eosinophilic cuticles and sebaceous glands are usually present in the cyst wall [2]

It is reported that early-onset steatocystoma multiplex is mostly caused by a mutation in KRT17 [3]. Steatocystoma multiplex most often occurs on the chest, neck, axillae and proximal extremities where the pilosebaceous apparatus is well developed, but it rarely occurs on the scalp. Steatocystoma multiplex limited to the scalp was first described by Marley et al. in 1981 [4]. Since then, there have been several similar reports of its occurrence on the scalp. Our case is unique due to the late age of onset and the characteristics of multiple lesions. In contrast to the typical steatocystoma multiplex, cases limited to the scalp are usually nonhereditary and do not develop until later in life, as in our case. It has been reported that steatocystoma multiplex may be associated with other abnormalities, such as pachyonychia congenital, congenital alopecia, hypohidrosis, hypothyroidism and acrokeratosis verruciformis of Hopf [5]. However, in our case, there were no associated accompanying symptoms. The clinical differential diagnosis of steatocystoma multiplex

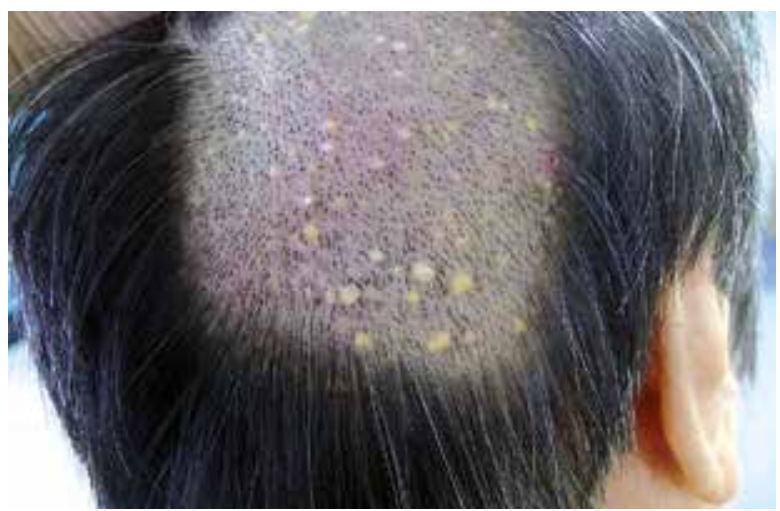

Figure 1. Multiple white and yellow cystic nodules scattered on the scalp

Address for correspondence: Xu Chen, Heng Gu, Institute of Dermatology, Jiangsu Key Laboratory of Molecular Biology for Skin Diseases and STIs, Chinese Academy of Medical Science and Peking Union Medical College, 12 Jiangwangmiao St, Nanjing 210042, China, phone: +86 258547 8919, fax: +86 25 85414477, e-mail: chenx@pumcderm.cams.cn, guheng@pumcderm.cams.cn Received: 21.08.2018, accepted: 12.09.2018. 


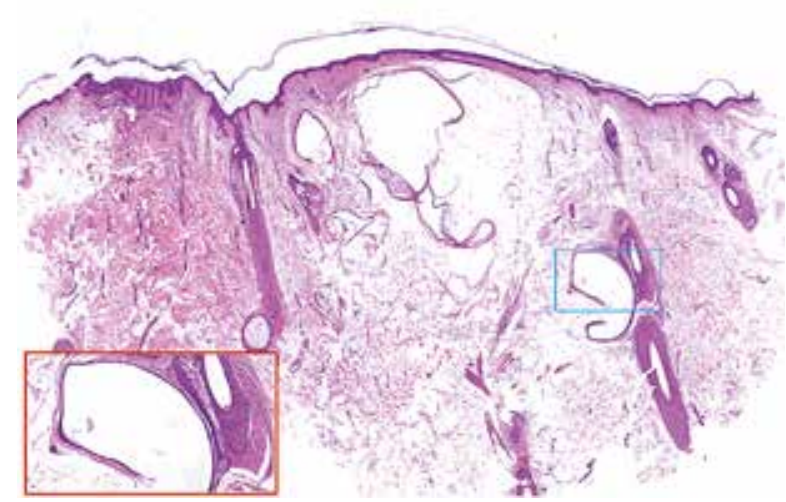

Figure 2. Histopathological examination showed multiple epithelial cysts in the dermis. The folded cystic wall was lined with stratified squamous epithelium without a granular layer. Sebaceous glands were lying close to the cyst wall $(H+E, 10 x$; inset 100x)

includes eruptive vellus hair cyst, epidermal cysts, syringoma, xanthomatosis and Fordyce disease, which may present with multiple, dome-shaped, scattered whiteto-yellow papules, nodules or cysts. When the lesions are inflamed, folliculitis and hidradenitis suppurativa should be identified. Biopsy can be done to make a definite diagnosis and exclude other possibilities according to respective histological features. Based on the age of onset, no family history, clinical features of the lesions and histopathological features of dermal cysts without a granular layer, our case accords with the diagnosis of late onset steatocystoma multiplex. There are many ways for treatment of this disease which can be selected based on the location, size, and number of lesions. Generally, steatocystoma multiplex does not need to be treated. For a certain purpose, cryotherapy, erbium:YAG or $\mathrm{CO}_{2}$ laser therapy, needle aspiration, oral isotretinoin and surgery are potential therapeutic options that may be used in isolated form or in combination [6]. For inflamed lesions, it has been reported to respond well to oral retinoid treatment [7]. In our case, considering the multiple lesions, its benign condition and lack of obvious symptoms, our patient declined further treatment after a detailed explanation of the illness. At present, the patient is still being followed up and the disease remains stable and shows no progress.

\section{Acknowledgments}

Meng Jiang and Mengli Zhang equally contributed to this article.

This work was supported by grants from the National Natural Science Foundation of China (No. 81773342, 81673083, 81703153), CAMS Innovation Fund for Medical Sciences (CIFMS-2017-I2M-1-017 and 2016-12M-1-005), PhD Programs Foundation of Ministry of Education of China (No. 20131106120046), the Jiangsu Provincial Spe- cial Program of Medical Science (BL2012003), the Open Foundation of the State Key Laboratory of Pollution Control and Resource Reuse (PCRRF17032) and PUMC Youth Fund and Fundamental Research Funds for the Central Universities (3332014008).

\section{Conflict of interest}

The authors declare no conflict of interest.

\section{References}

1. Plewig G, Wolff HH, Braun-Falco O. Steatocystoma multiplex: anatomic reevaluation, electron microscopy, and autoradiography. Arch Dermatol Res 1982; 272: 363-80.

2. Cho S, Chang SE, Choi JH, et al. Clinical and histologic features of 64 cases of steatocystoma multiplex. I Dermatol 2002; 29: 152-6.

3. Gass JK, Wilson NJ, Smith FJ, et al. Steatocystoma multiplex, oligodontia and partial persistent primary dentition associated with a novel keratin 17 mutation. Br J Dermatol 2009; 161: 1396-8.

4. Marley WM, Buntin DM, Chesney TM. Steatocystoma multiplex limited to the scalp. Arch Dermatol 1981; 117: 673-4.

5. Cole LA. Steatocystoma multiplex. Arch Dermatol 1976; 112: 1437-9.

6. Mortazavi H, Taheri A, Mansoori P, et al. Localized forms of steatocystoma multiplex: case report and review of the literature. Dermatol Online J 2005; 11: 22.

7. Düzova AN, Sentürk GB. Suggestion for the treatment of steatocystoma multiplex located exclusively on the face. Int J Dermatol 2004; 43: 60-2. 